\section{[gw22-e0937] ESSENTIAL ROLE OF CONNECXIN 43 IN ISCHAEMIC POSTCONDITIONING DURING MYOCARDIAL ISCHAEMIA-REPERFUSION}

Nan Li, Yu Jie Zeng Department of Cardiology, Japan Friendship Hospital, China

10.1136/heartjnl-2011-300867.416

Background Recent study show that ischaemic postconditioning (IPC) may provide a new therapy for reperfusion after myocardial infarction. To evaluate the cardio protection of IPC during myocardial ischaemia-reperfusion by measuring the arrhythmia, $\mathrm{CK}, \mathrm{LDH}, \mathrm{MDA}, \mathrm{NO}$, size of infarction, the distribution, amount and Phosphorylation state of connexin 43 in IPC during myocardial ischaemia-reperfusion by intervented of mito-K $\mathrm{K}^{+}$ATP selected opener diazoxide and selected blocker 5-hydroxydecanoic acid (5-HD.

Discussion The effect of $\mathrm{Cx} 43$ on IPC and relationship between $\mathrm{Cx} 43$ and mito-K+ $\mathrm{K}^{+}$TTP.

Methods Male adult SD rats were randomly divided into five different groups: sham-operation; ischaemia-reperfusion (IR); ischaemia Postconditioning (IPC), diazoxide+IR and 5 -HD+IPC. Observed the incident rate of arrhythmia, measured the level of Plasma CK, LDH, MDA and NO. Ischaemic size and Infarct size were measured by Evans blue and TTC staining respectively. The distribution of connexin 43 on cardiac myocytes were determined by immunohistochemical stains by light-microscopy. The amount and Phosphorylation state of connexin 43 were determined by western blotting.

Results The arrhythmia incident rate was $60 \%, 25 \%, 35 \%$, $45 \%$ in IR group, IPC group, diazoxide group and 5 -HD group, respectively. Infarct size was smaller in IPC group and diazoxide group than in IR group. The level of Plasma CK, LDH, MDA and NO were decreased by IPC and diazoxide $(p<0.05)$, and the level was similar in IPC group and diazoxide group. Immunohistochemistry: The results of this experiments indicate an altered distribution of $\mathrm{Cx} 43$, which is displayed as a spreading of its original positions in gap junctions. Intensity of $\mathrm{Cx} 43$ in the lateral sarcolemma was more higher than in terminal intercalated disks of the cell in IR group and 5-HD group, indicated $\mathrm{Cx} 43$ occurred redistributed. IPC can reduced the redistribution. Western blot: the amount of total Cx43 and $\mathrm{P}-\mathrm{Cx} 43$ was significantly decreased in IR group in myocytes reperfusion for $1 \mathrm{~h}$ and $3 \mathrm{~h}(\mathrm{p}<0.05)$. The mount of $\mathrm{P}-\mathrm{Cx} 43$ in IPC group was less than sham-operation group after reperfusion, but with the prolong of reperfusion were increased $(\mathrm{p}<0.05)$. Compared with IPC group, the amount of P-Cx43 was decreased in 5-HD+IPC group after $1 \mathrm{~h}$ and $3 \mathrm{~h}$ reperfu$\operatorname{sion}(\mathrm{p}<0.05)$.

Conclusions IPC can significantly reduce the ischaemiareperfusion injury. IPC can preserved $\mathrm{P}-\mathrm{Cx} 43$ during ischaemia reperfusion injury. Mito- $\mathrm{K}^{+} \mathrm{ATP}$ Play a important role in 
reduce degradation of $\mathrm{Cx} 43$, the mechanism of which may be related with the opened mito- $\mathrm{K}^{+}$ATP. 